\title{
Raising argument strength using negative evidence: a constraint on models of induction
}

\author{
Daniel Heussen • Wouter Voorspoels • \\ Steven Verheyen • Gert Storms • James A. Hampton
}

Published online: 21 May 2011

(C) Psychonomic Society, Inc. 2011

\begin{abstract}
Both intuitively, and according to similaritybased theories of induction, relevant evidence raises argument strength when it is positive and lowers it when it is negative. In three experiments, we tested the hypothesis that argument strength can actually increase when negative evidence is introduced. Two kinds of argument were compared through forced choice or sequential evaluation: single positive arguments (e.g., "Shostakovich's music causes alpha waves in the brain; therefore, Bach's music causes alpha waves in the brain") and double mixed arguments (e.g., "Shostakovich's music causes alpha waves in the brain, X's music DOES NOT; therefore, Bach's music causes alpha waves in the brain"). Negative evidence in the second premise lowered credence when it applied to an item X from the same subcategory (e.g., Haydn) and raised it when it applied to a different subcategory (e.g., AC/DC). The results constitute a new constraint on models of induction.
\end{abstract}

Keywords Induction · Negative evidence · Categories · Models of induction

Much of everyday reasoning consists of inductive inference, in which induction is taken in its broadest sense as an inference to an uncertain conclusion. One strategy to make

D. Heussen $(\varangle) \cdot$ W. Voorspoels $\cdot$ S. Verheyen $\cdot$ G. Storms

Psychology Department, University of Leuven,

Tiensestraat 102,

3000 Leuven, Belgium

e-mail: daniel.heussen@psy.kuleuven.be

\section{J. A. Hampton}

Psychology Department, City University,

Northampton Square,

London, UK such an inference is to use past experiences - for example, "My car has always started, so it's reliable and will start today." Another strategy is to use category membershipfor example, "My car is a German car, so it's reliable and will start today." Research on induction in psychology has predominately focused on the latter of the two. In a typical experiment, people are told that one or several categories have a particular property and are asked to extend that property to other categories within a given domain. Participants, for instance, might be asked to judge how likely it is that bobcats use serotonin as neurotransmitter given that both tigers and cougars do (Smith, Shafir, \& Osherson 1993). Numerous phenomena relating to category-based property induction have been documented (e.g., Hampton \& Cannon, 2004; Heit, Hahn, \& Feeney 2004; Rips, 1975; for a review, see Heit, 2000), and various models have been proposed to account for people's judgments of inductive arguments such as these (e.g., Blok, Medin, \& Osherson 2007; Heit, 1998; Kemp \& Tenenbaum, 2009; Medin, Coley, Storms, \& Hayes 2003; Osherson, Smith, Wilkie, Lopez, \& Shafir 1990; Sloman, 1993).

In the majority of cases, experimental work and modeling efforts have focused on arguments involving positive evidence-premises that state that some entity possesses the to-be-projected property. In reality, though, we do not only receive positive evidence for our hypotheses. We are often confronted with negative evidence, evidence that states that some entity of the same or of a similar category DOES NOT possess the to-be-projected property. For instance, in evaluating whether bobcats use serotonin as neurotransmitter, we might find out that tigers do, but cougars do not. How do we integrate the negative evidence, and how does it influence our judgment about bobcats? 
In the present article, we are interested in the influence of negative evidence on argument strength. More precisely, we are interested in the direction of this influence. Contrary to intuition and to the general predictions of similarity-based theories of induction, we present evidence that, under certain circumstances, negative evidence can actually facilitate the generalization of a property. In what follows, we will identify and discuss in more detail the general assumption that negative evidence has a negative effect on argument strength. Then, we will present three experiments that undermine the universal validity of this assumption. In the General Discussion section, we address in more detail the implications of these findings for well-known models of induction.

\section{Monotonicity in inductive reasoning}

In absence of other information, it is generally assumed that generalization relies on similarity (Blok et al., 2007; Osherson et al., 1990; Rips, 1975; Shepard, 1987; Sloman, 1993; Smith et al., 1993). In the context of category-based property induction, similarity between premise and conclusion categories determines the influence that a premise has on argument strength: The more similar the premise and conclusion categories are, the greater the influence of the evidence on the conclusion (e.g., Rips, 1975). In evaluating whether bobcats have property $\mathrm{x}$, knowing that tigers do seems to provide stronger evidence than discovering that penguins do. Moreover, this relation between similarity and arguments has been shown to hold for additional premisesdiscovering that not only tigers have property $\mathrm{x}$, but also lions and domestic cats, will further strengthen one's belief that indeed bobcats too must have the property. The positive relation between the number of positive premises and argument strength has been referred to as monotonicity in inductive reasoning and is supported by many empirical results (e.g., Feeney, 2007; McDonald, Samuels, \& Rispoli 1996; Osherson et al., 1990, Osherson, Stern, Wilkie, Stob, \& Smith 1991; for summaries, see Hayes, Heit, \& Swendsen 2010; Heit, 2000). ${ }^{1}$

Now, what can we expect when negative evidence comes into play? It is important to realize that there is no reason to consider not having a property as essentially different from having a property. ${ }^{2}$ In other words, discovering that lions DO NOT have property $\mathrm{x}$ provides

\footnotetext{
${ }^{1}$ We are aware that for positive evidence, there are at least two notable exceptions to monotonicity reported in the literature (Medin et al., 2003; Osherson et al., 1990). However, reviewing the literature reveals that these exceptions are what they are-exceptions. We'll address them further below.

${ }^{2}$ In fact, rather than using explicit negation, negative evidence is sometimes implemented as having a contrasting type of property (e.g., Kalish \& Lawson, 2007).
}

a reasonable basis to conclude that bobcats DO NOT have $\mathrm{x}$, analogously to having a property. Obviously, the conclusion that bobcats DO NOT have $\mathrm{x}$ is inversely related to the conclusion that bobcats have $\mathrm{x}$. Hence, bobcats having $\mathrm{x}$ becomes less likely in the light of lions NOT having $x$. Following a similarity-based approach, negative evidence about a similar entity, therefore, decreases argument strength regarding a positive conclusion in line with monotonicity. What research on negative evidence exists supports this general principle of monotonicity (e.g., Blok et al., 2007; Heussen \& Hampton, 2011; Osherson et al., 1991).

In short, evidence should raise argument strength if it is positive (i.e., when it states that some entity has the property) and should lower it if it is negative (i.e., when it states that some entity does not have the property), with similarity determining the size of the change in argument strength. Argument strength should move in the same direction as the "sign" of the evidence. In line with previous research, we call this the Monotonicity Principle. To the extent that theories of inductive reasoning rely on similarity, models of induction endorse the Monotonicity Principle (e.g., Blok et al., 2007; Osherson et al., 1990; Sloman, 1993). Models can, however, have other mechanisms that may explain violations of the principle (e.g., Osherson et al., 1990); this will be considered in more detail in the General Discussion section.

\section{In search of evidence against monotonicity}

On the side of positive evidence, there are some notable violations to the principle (Feeney, Coley, \& Crisp 2010; Medin et al., 2003; Osherson et al., 1990). These violations of monotonicity are based on using additional evidence that comes from a category that is different from the one that includes both the original premise and conclusion categories. For example, Osherson et al. reported that a greater proportion of people preferred an argument from fly to bee than from fly and orangutan to bee. Adding positive evidence from a category (mammals) different from that of the conclusion and first premise (insects) elicited a drop in argument strength, thus disconfirming the monotonocity principle for positive evidence. A related phenomenon has been reported by Medin et al., who showed that people judged an argument from polar bear to penguin as stronger than the argument from polar bear and brown bear to penguin. Again, adding positive evidence decreased the argument strength, contrary to the Monotonicity Principle. However, in this case, the violation was the result of the addition of positive evidence from a more specific category (bears) than from the category including the first premise and the conclusion (animals). 
The symmetry between positive and negative evidence suggests that similar violations of the Monotonicity Principle should be found for negative evidence. Moreover, there is evidence from developmental studies that suggests that negative evidence (or, more precisely, contrastive information) can have a beneficial effect on generalizations, at least among children (Kalish \& Lawson, 2007; Waxman, Lynch, Casey, \& Baer 1997). However, because these studies departed from the standard paradigm in (a) using arguments about individuals rather than whole classes, (b) using implicit rather than explicit negation, and because the results for adults were equivocal, it is unclear to what extent these findings may generalize to a standard category-based property induction paradigm within an adult population.

In the present study, we aimed to establish nonmonotonicity effects in a standard category-based induction task when adding negative evidence to an argument. We do not aim to offer a definitive theoretical account of how or why inductive strength can increase with negative evidence, nor are we making a universal claim about the circumstances or conditions in which the effect occurs. Rather, our initial aim is to make an existential claim that there are cases in which argument strength can be strengthened by negative evidence.

Across three experiments, we used a category-based property induction paradigm with blank properties - properties that participants are likely to have very little knowledge about. Participants saw single positive (e.g., "Given that freight ships create conversion currents, how likely is it that cruise ships do so?") and double mixed premise arguments (e.g., "Given that freight ships create conversion currents, and that hovercraft ships DO NOT, how likely is it that cruise ships do so?"). They either made forced choice judgments between the arguments (Experiments 1 and 2) or they sequentially evaluated them (Experiment 3).

In these arguments, the first premise and conclusion were always from the same subcategory (e.g., freight ships and cruise ships). The second (negative) premise used either another item from the same subcategory (e.g., ferry ships), or an item from a different subcategory (e.g., hovercrafts).

Our underlying hypothesis for the three experiments was that a preference for arguments containing negative evidence (i.e., a preference for mixed over single premise arguments) will be more likely when negative evidence is instantiated by an item from a contrasting subcategory than by those of the positive premise and the conclusion. In contrast, if the negative evidence is instantiated by an item from the same subcategory, the usual negative impact on argument strength is expected.

\section{Experiment 1}

In Experiment 1, participants were asked to choose the stronger of two arguments, one with a single positive premise and the identical argument with an additional negative premise.

Shostakovich elicits alpha waves.

Shostakovich elicits alpha waves.

Bach elicits alpha waves.

Music of AC/DC does not.

Bach elicits alpha waves.

If the Monotonicity Principle is true, adding a negative premise to an argument with a single positive premise should, in principle, not increase argument strength. Hence, participants should always choose the single positive premise. Selecting a mixed premise argument as stronger indicates a violation of the Monotonicity Principle.

\section{Method}

Participants Participants were 32 first-year undergraduate students at the University of Leuven who each completed a booklet for course credit.

Design In a repeated measures design, participants made forced choice judgments about a list of 30 pairs of arguments. Participants were instructed to assume that the premises of the arguments are stating facts and were asked to make a forced choice for the argument whose premises provide better reasons to belief the conclusion. Each pair consisted of a single and a mixed premise argument. The mixed premise argument was identical to the single premise argument with the exception of an added negative premise. Half of the pairs were target pairs, and half were control pairs. Two random orders of items were used.

Materials The premises and the conclusion of each argument contained exemplars from a single category (e.g., insects, fruit, wines, car companies). For the target items, the positive premise and the conclusion were from one loosely defined subcategory (e.g., flying insects, tropical fruit, European wines, German car companies), whereas the negative premise was an exemplar from a contrasting subcategory (e.g., crawling insects, Northern European fruit, New World wines, Italian car companies). The negative evidence in the control items came from the same subcategory as for the positive premise and conclusion. The selection of items from loosely defined subcategories was based on the first two authors' intuitions. $^{3}$ The properties used in the arguments were

\footnotetext{
${ }^{3}$ In order to test the authors' intuition, we asked a set of participants to perform a grouping task. Participants circled the two exemplars out of the triplet present in the argument that belong together. For the target condition, 10 out of 15 items had the majority of participants (around .8 and above) select the first premise and conclusion as the grouped pair (overall average .77 for the target condition). In contrast, across items the average proportion of people selecting the first premise and conclusion for the control condition was less than 5 .
} 
realistic characteristics that participants were likely to have very little knowledge about (e.g., produce ocytoncine; have mitochondrion in their cells; create a conversion current). A list of the items used in all three experiments can be found in the Appendix.

Procedure Students participated in groups and completed the questionnaire as part of a series of tasks. The task took no more than $5 \mathrm{~min}$.

\section{Results}

Figure 1 shows the average proportion of responses across 15 target and 15 control items that showed a preference for the mixed premise argument containing negative evidence over the single positive premise argument.

An independent sample $t$ test on the average proportion of participants, $t(28)=6.4, p<.001$, and a paired-sample $t$ test on the average proportion of items, $t(31)=6.5, p<.001$, showed a significant difference, $\min F^{\prime}(1,59)=20.8, p<.01$. between our target and control items in response preference for arguments involving negative evidence, with both proportions being significantly higher than zero [control, $\min F^{\prime}(1,43)=28.9, p<.01 ;$ target, $\min F^{\prime}(1,35)=74.5$, $p<.01]$. Participants were two and a half times more likely to select the argument containing negative evidence as stronger among target items than among control items. Preference for negative evidence arguments rose from just under $20 \%$ in the control items to nearly $50 \%$ in the target items.

\section{Discussion}

In Experiment 1, we demonstrated in a forced choice paradigm that participants can be tempted into preferring an argument that contains negative evidence to one with just

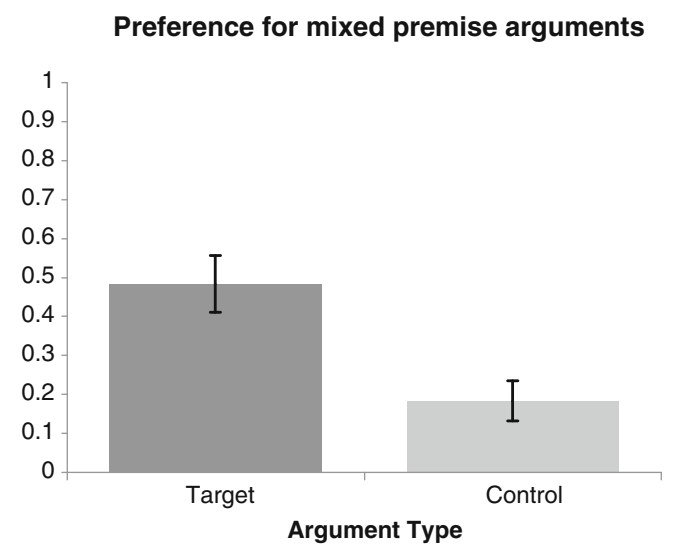

Fig. 1 Average proportion of responses selecting the mixed premise argument for target and control items. Error bars constitute $95 \%$ confidence intervals calculated on the participant data a single positive premise. Although this preference was significantly greater when the negative evidence was instantiated by an item from a different rather than from the same subcategory (reflecting our manipulation), the preference for mixed premise arguments did not rise above $50 \%$ on average.

What does a response pattern of $50 \%$ represent? One could argue that this proportion reflects responses at chance level. For the target items, participants may have perceived the strength of single and mixed premise arguments as identical, hence making a choice according to a coin flip. If this is the case, the present finding does not support the conclusion that adding negative evidence can increase argument strength, but merely shows that there are cases in which negative evidence does not lower argument strength. Note, however, that, following the Monotonicity Principle, this implies that the negative premise concerns a category that is irrelevant-otherwise it would lower argument strength. We believe that this is not the case.

In order to construct the target items, we chose categories that were explicitly similar in a very salient respect (belonging to the same superordinate category, e.g., music). Consequently, these categories bear relevance to the arguments in question, and following the Monotonicity Principle, should always lower argument strength. This prediction is contradicted by the data: Participants were clearly tempted to choose the argument that included negative evidence at a rate significantly above zero (the prediction of the Monotonicity Principle), and significantly more than the control items (which form an empirical baseline). Thus, we believe that the response pattern of .5 actually reflects a degree of deviation from the preference for single premise arguments.

In Experiment 2, we tried to support this by comparing our target items with items that contain irrelevant negative evidence. If the preference pattern for the irrelevant condition turns out to be the same as that for our target items, then our data do not show a violation of the Monotonicity Principle. However, if we do find a significant difference, the response pattern of .5 cannot represent chance level responding and hence demonstrates an increase in argument strength through the presence of negative evidence.

The following two studies aimed to back this claim by contrasting relevant with irrelevant negative evidence (Experiment 2) and by explicitly demonstrating an increase in rated argument strength from single to mixed premise arguments within participants (Experiment 3).

\section{Experiment 2}

In Experiment 1, we used a within-subjects design with target and control condition being instantiated by differ- 
ent items. In Experiment 2, we replicated the study using a between-subjects design in order to test whether the manipulation to elicit the effect is robust within items. As before, participants were asked to choose between a single premise argument and a mixed premise argument (containing a negative premise). Different groups of participants were presented with the same positive premise and conclusion, but with different negative evidence premises that either came from a subcategory different from the positive premise and the conclusion (target), or from the same subcategory (control). In addition, we added a third condition, in which the additional evidence was negative but irrelevant (irrelevant):

Shostakovich's music elicits alpha waves.

A falling rock does not elicit alpha waves.

Bach's music elicits alpha waves.

The irrelevant condition allows us to test whether responses to the target condition in Experiment 1 constituted chance level responding. Chance level responding in the target condition implies that the negative evidence is irrelevant for the conclusion. If the preference for arguments containing negative evidence in the irrelevant condition of Experiment 2 can be shown to be significantly lower than in the target condition, then responses to the target condition - even if they are at .5-can not constitute chance level responding and hence indicate a rise in argument strength.

Responses for the control items in Experiment 1 were not at normative levels, with nearly $20 \%$ of responses on average across participants violating the Monotonicity Principle. Under the assumption of the Monotonicity Principle, participants would consistently choose the single premise argument, resulting in no legitimate variation in response pattern. Participants may hence have been somewhat coerced into indicating a preference for the mixed premise argument, at least for a few of the control items. In Experiment 2, we introduce thirty filler items that elicit all possible response patterns. Fillers remained the same across the three conditions.

\section{Method}

Participants Participants were 121 first-year undergraduate students at the University of Leuven who each completed a booklet for course credit.

Design and materials The task was identical to that in Experiment 1. Participants were asked to judge which of two arguments (a single and a mixed premise) provides better reasons to belief the conclusion. A between-subjects design was used in which 10 of the target items in Experiment 1 (target items that showed the strongest effect) were presented in three different conditions - target, control, and irrelevant. Participants were randomly allocated to one of the three conditions, with roughly 40 participants in each condition. As in the previous experiment, the target and the control condition contained negative evidence from a different or from the same subcategory as the positive premise and the conclusion, respectively. In addition, an "irrelevant" condition presented negative evidence in the form of an exemplar from a superordinate category different than that of the positive premise and the conclusion.

The same thirty filler items were used across the three conditions. These consisted of 10 purely positive argument pairs (e.g., "Lions have enzyme $x$, tigers have enzyme $x$. How likely is it that cheetahs have enzyme $x$ ?") that should clearly elicit a preference for the mixed premise argument in order to provide legitimate variation in response choices and 20 argument pairs with negative evidence that should elicit a preference for the single premise argument in a form similar to our control items.

Procedure Students participated in groups and completed the questionnaire as part of a series of tasks. The task took no more than $8 \mathrm{~min}$.

\section{Results and discussion}

In line with Experiment 1, Fig. 2 shows the average proportion of responses that indicate a preference for mixed premise arguments containing negative evidence over single positive premise arguments.

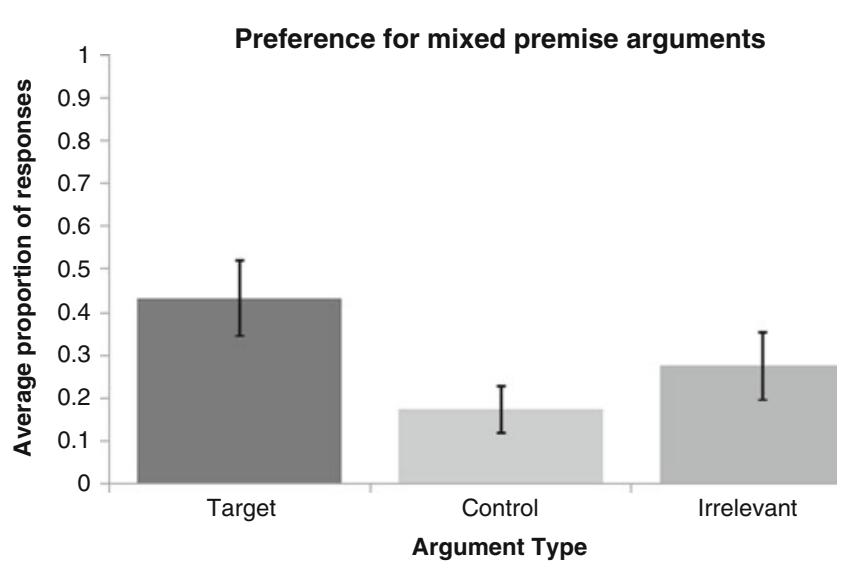

Fig. 2 Average proportion of responses selecting the mixed premise argument for target, control, and irrelevant items. Error bars constitute $95 \%$ confidence intervals calculated on the participant data 
A one-way ANOVA across participants and items showed a significant difference in preference for mixed premise arguments between conditions, $\min F^{\prime}(2,89)=7.53$, $p<.001$. A planned pairwise comparison for participants and items showed a significant difference between the target and control condition, $\min F^{\prime}(1,42)=14.35, p<.001$, and between target and irrelevant, $\min F^{\prime}(1,65)=4.87, p<.05$, but not between irrelevant and control, $\min F^{\prime}(1,48)=2.79$, $p=.102$.

In Experiment 2, we thus replicated the findings of Experiment 1 in a between-subjects design, providing evidence that participants violate the Monotonicity Principle. The same single-premise arguments were either preferred or rejected, depending on the additional negative evidence. People showed a greater preference for arguments containing negative evidence when the evidence came from a different rather than from the same subcategory as the positive premise and the conclusion. Furthermore, using irrelevant negative evidence from an unrelated superordinate category resulted in responses closer to the control than to the target condition, indicating that irrelevant evidence in a forced choice paradigm leads to a preference for single premise arguments and not coin-flip responding. If the $50 \%$ choice for the target arguments in Experiment 1 had simply reflected a view that the negative premise was irrelevant, then we should have observed a similar level of choice for the irrelevant condition in Experiment 2. The significant difference between these two conditions therefore rules out this account.

\section{Experiment 3}

In order to explore the observed violation of the Monotonicity Principle further, Experiment 3 was designed to replicate the effect in a new paradigm. We changed our procedure from a forced choice to a sequential judgment task. Participants first evaluated the single premise argument, then received the negative premise and evaluated the mixed premise argument.

Contrary to the Monotonicity Principle, we predict an increase in argument strength from single to mixed premise arguments for the target items but not for the control items. The control items should reflect a clear drop in argument strength, in line with the Monotonicity Principle. Note that in the present experiment, we measured the effect within a person and an item, the strongest test for the claim that negative evidence can indeed raise argument strength. In addition, people were able to express the view that the strength was unaffected by giving the same rating to each argument.

\section{Method}

Participants Fourteen undergraduate students at the University of Leuven participated for course credit.

Design and materials A repeated measures design was used to measure the change in argument strength from single to mixed premise arguments. Participants were asked to judge both single and mixed premise arguments as well as whether argument strength had decreased, stayed unchanged, or had increased between the first and second judgment. Judgments of argument strength were measured on an 11-point scale. Two random orders of items were used.

The arguments were identical to those used in Experiment 1. The arguments were embedded in little vignettes describing the first premise as a well-established scientific or specialist fact and then asking participants to evaluate the conclusion. Participants then received a second piece of information again described as a well-established fact, and were asked to evaluate whether this lowered, raised, or left their judgment of the conclusion unchanged. Subsequently, they gave a final judgment of the conclusion, given both facts.

Procedure Participants completed the booklets within an individual testing session.

\section{Results and discussion}

Figure 3 shows the average proportion of responses indicating a decrease, no change, or increase in argument strength from the first to the second judgment for target and control items.

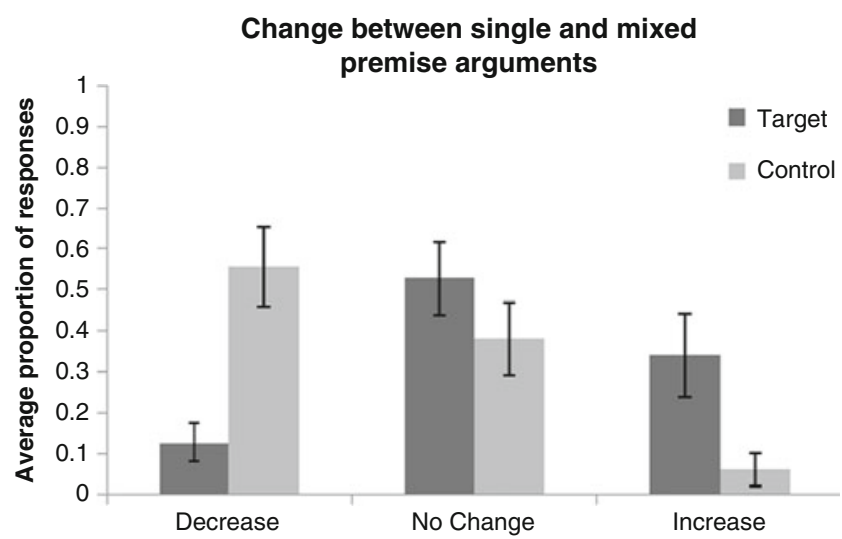

Fig. 3 Average proportion of responses indicating a change in argument strength from the single positive (first judgment) to mixed premise arguments (second judgment) for both control and target items. Error bars constitute $95 \%$ confidence intervals calculated on the participant data 
A $2 \times 2$ ANOVA $^{4}$ for participants and items revealed a significant interaction between the direction of change in people's judgment (i.e., decrease, increase) and item type, $\min F^{\prime}(1,39)=45.3, p<.001$. For the target items, a greater average proportion of responses across participants and items indicated an increase rather than a decrease in argument strength from the first to the second judgment, $\min F^{\prime}(1,26)=5.8, p<.05$, whereas for the control items, the reverse pattern was found, $\min F^{\prime}(1,26)=37.5, p<.001$. Although for the target items the greatest proportion of items did not change in strength, indicating that negative evidence for those items was perceived as irrelevant, the average proportion of responses indicating an increase was significantly larger for the target items than for the control items (Target: $M=0.34, S D=0.19$; Control: $M=0.06$, $S D=0.07), \min F^{\prime}(1,36)=22.7, p<.001$.

This pattern of results was also reflected in the ratings of the single and mixed premise arguments. Figure 4 shows the average argument strength of single positive and mixed premise arguments for target and control items.

A $2 \times 2$ ANOVA for participants and items revealed a significant interaction between the type of argument (levels: single premise vs. mixed premise arguments) and item type (levels: target vs. control items), $\min F^{\prime}(1,39)=38.8$, $p<.001$. Target items showed a significant increase in argument strength from single to mixed premise arguments, $\min F^{\prime}(1,26)=7.0, p<.05$, whereas control items showed a significant decrease, $\min F^{\prime}(1,25)=33.9, p<.001$. Among the single premise arguments, target and control items did not show a significant difference in argument strength [item, $F(1,13)=6.2, p<.05$; participant, $F(1,28)=2.7$, $\left.p=.114 ; \min F^{\prime}(1,41)=1.9, p=0.178\right]$. These results confirm our contention that it is possible to increase argument strength by introducing negative evidence.

In Experiment 3, we tested the Monotonicity Principle in a sequential evaluation task within items and participants; hence, it constitutes the most stringent of the three studies. Although the majority of people indicated no change in ratings of our target items between the first and the second judgment, a significant proportion did indicate an increase. ${ }^{5}$ If the results of Experiments 1 and 2 constituted coin-flip responding on the grounds that the negative premise was irrelevant and did not change the argument, here we should have seen very few responses indicating the increase option, and almost all responses indicating the no change option. The results of Experiment 3 do not support this view. To the contrary, in line with the proportions, average ratings of the

\footnotetext{
${ }^{4}$ The no-change responses were omitted from the analyses to avoid violations of the independence assumption of ANOVA.

${ }^{5}$ Although we restrict ourselves in referring to the effect on people's preferences, the effect held across both people and items as indicated by the $\min F^{\prime}$ analyses.
}

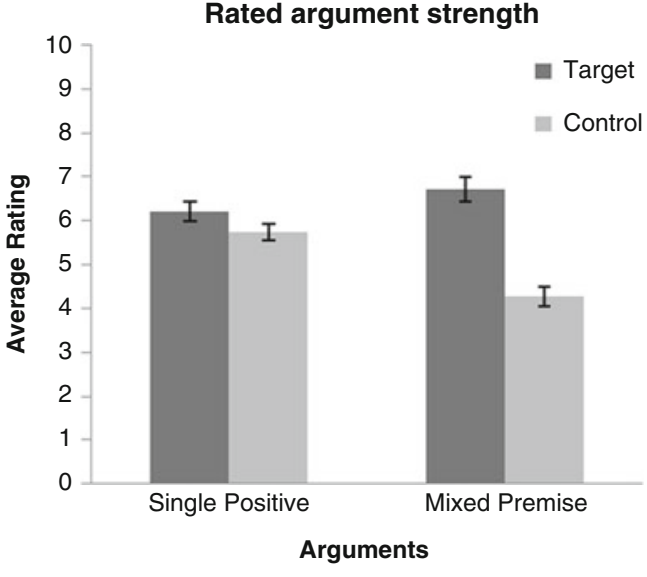

Fig. 4 Average argument strength of single positive (first judgment) and mixed premise arguments (second judgment). Error bars constitute $95 \%$ confidence intervals calculated on the participant data

target items went up significantly from the single to the mixed premise arguments, providing clear evidence for a rise in argument strength. In contrast, the control items showed a substantial decrease in argument strength.

\section{General discussion}

Imagine you want to test the proposition that "all ravens are black." There are two related hypotheses that need to hold for the proposition to be true. One is "If it's a raven, then it is black." The second is, "If it's not black, then it's not a raven." Intuitively, seeing something that is a raven and that is black confirms the hypothesis, "If it's a raven, then it is black." But, similarly, seeing something that is not black and not a raven confirms the hypothesis, "If it's not black, then it's not a raven." Hempel (1945) demonstrated the logical equivalence of these two hypotheses, showing that evidence that supports one hypothesis logically provides support for the other. Hence, according to formal logic, encountering a "white swan" is confirmatory evidence for the proposition, "All ravens are black." But, as Hempel argued, and most readers would agree, this seems counterintuitive, and hence poses a paradox between formal logic and intuition.

Hempel's (1945) paradox depends on what we called the Monotonicity Principle of the influence of evidence. In the present article, however, we have demonstrated that it is possible to raise argument strength by providing negative evidence. There are arguments in which, figuratively speaking, "seeing a white swan" does provide evidence for the conclusion, "all ravens are black." Although this finding does undermine the paradox, as such, it does not eliminate the paradox. According to logic, any non-black non-raven, including pink sunsets, provides support for the conclusion "all ravens are black," and that is clearly not the 
case. More modestly, our finding highlights the need for an account that is able to differentiate between negative evidence that lowers and negative evidence that raises credence in the conclusion of an argument. Such an account would provide a psychological demarcation of Hempel's paradox.

\section{Models of induction and nonmonotonicity}

The present findings not only have implications for Hempel's (1945) paradox but also raise an important question concerning existing models of induction-namely, how to incorporate the influence of negative evidence. To date, most models of induction have focused on the influence of positive evidence (e.g., Osherson et al., 1990; Rips, 1975; Sloman, 1993), with only two models providing an explicit formulation of the influence of negative evidence (Blok et al., 2007; Kemp \& Tenenbaum, 2009). In order to provide some discussion of the main models, we'll make minimal assumptions where necessary on how negative evidence could be implemented. Although an elaborate presentation of the models and possible adaptations to account for our results falls outside the scope of this contribution, a model-based analysis of the present finding can lead to a better understanding of the phenomenon.

\section{Feature-based induction model}

The feature-based induction model (FBIM; Sloman, 1993) derives the strength of a conclusion from the association between existing and novel features that is built from the evidence in the premises. Generalization therefore increases as similarity increases. Although FBIM is not formulated for negative evidence, assuming the symmetry of having-aproperty and not-having-a-property, the model's predictions are in line with the Monotonicity Principle and hence cannot handle our findings.

\section{SimProb}

In the SimProb model (Blok et al., 2007), a judgment of argument strength is conceptualized as a conditional probability judgment that relates the prior probability of a conclusion to the relevance of the evidence (by degree of similarity) and the degree to which the evidence is surprising (by prior probability of the evidence). The SimProb model explicitly allows for negative evidence premises, yet does not allow a rise in argument strength following the addition of a negative premise. According to the SimProb model, relevant evidence will raise argument strength if it is positive and lower argument strength if it is negative. As such, the model endorses the Monotonicity Principle and hence cannot handle the present findings.

\section{Similarity-coverage model}

The well-known similarity-coverage model (SimCov; Osherson et al., 1990) relates the strength of an argument to two similarity-based components. First, the similarity between the premise and conclusion categories plays an important role. In its present formulation, the model does not incorporate negative evidence; however, assuming a symmetry between having-a-property and not-having-a-property leads to model predictions that are necessarily in line with the Monotonicity Principle (e.g., Osherson et al., 1991). In other words, the similarity term cannot accommodate a raise in argument strength due to negative evidence. ${ }^{6}$

The second component that can influence argument strength according to the SimCov model is the coverage term, which reflects the extent to which an immediately relevant superordinate category is "covered" by the premise categories. Coverage is operationalized by computing the average maximum similarity of the premise categories to other members of the nearest superordinate category that includes premises and conclusion. Interestingly, the coverage component is crucial in explaining nonmonotonicity effects for strictly positive premises (Osherson et al., 1990), due to changes in the relevant superordinate category for the argument (see the introduction).

If we assume that in the single premise arguments (e.g., Shostakovich $\rightarrow$ Bach) people rely on the broader basiclevel category (e.g., MUSIC) as the immediate superordinate in the coverage term, the addition of the negative premise (e.g, AC/DC does not have the property) forces a change of the-to-be-covered category to a lower subcategory (e.g., CLASSICAL MUSIC). The latter, more specific, category is clearly better covered by the positive premise category. Therefore, adding the negative evidence may actually raise argument strength because the positive premise provides greater coverage for the more specific subcategory (i.e., CLASSICAL MUSIC) as compared with the more general basic level category (i.e., MUSIC). As such, the observed effect can be considered a negative evidence variant of the nonmonotonicity effect described by Osherson et al. (1990).

\footnotetext{
${ }^{6}$ Furthermore, the similarity component uses the maximum similarity of all premises to the conclusion. In mixed premise arguments, the sign of the similarity component would hence depend on whether the positive or the negative premise is more similar to the conclusion, leading to unnatural patterns of data (Heussen, Voorspoels, \& Storms 2010).
} 
Note, however, that this assumes that the coverage term covers only the positive premise and conclusion categories and not the negative premise categories. Furthermore, according to SimCov, people turn to the most specific superordinate category that includes both premise and conclusion categories. It is therefore unclear why, in the case of negative evidence, the basic level (e.g., MUSIC) should be considered the to-be-covered category for single positive premise arguments. Moreover, even if changing the to-be-covered category was the mechanism to deal with negative evidence that raises argument strength, it is then unclear how the SimCov model would handle our control items, in which the negative evidence that is at the same hierarchical level as both the positive premise and the conclusion lowers argument strength.

\section{Bayesian approaches}

In the Bayesian approach to inductive reasoning, it is assumed that people make optimal inferences on the basis of prior hypotheses about the distribution of the novel feature and the evidence provided through the premises (e.g., Heit, 1998; Tenenbaum \& Griffiths, 2001). Every hypothesis about a novel feature can be formulated as the extension of the feature - that is, which categories have it and which categories do not. The prior probability of such a hypothesis reflects the prior belief that the corresponding feature extension is correct, relative to other hypotheses. As evidence is observed (the premise), the probability of the hypotheses is updated following Bayes' rule, and the probability that a specific category has the property is updated accordingly.

A Bayesian inference mechanism as such does not exclude the possibility that negative evidence should raise the strength of an argument, given the right set of prior probabilities for the relevant hypotheses. To raise argument strength with negative evidence, the priors of the hypotheses require a strong a priori clustering of the positive premise and the conclusion category. In other words, hypotheses that the feature extends only to the positive premise and conclusion categories and not to the negative premise should be likely a priori (i.e., before the premises are considered). Moreover, hypotheses that do not endorse this clustering should receive a low prior - for instance, hypotheses that all categories have the property or that only the positive premise category has it, or any combination of categories from the positive and negative set. Given these priors, the posterior probability that the conclusion category has the property can increase when considering the negative premise.

Another way of looking at it is to consider an exhaustive hypothesis space. For instance, for the "elicitation of alpha waves among music," one might have a set of hypotheses consisting of "noise in general elicits alpha waves," "only music does," "only classical music does," or "it only applies to Shostakovich." Negative evidence may help in reducing the number of hypotheses by explicitly contradicting some of them (e.g., not all music elicits alpha waves). Furthermore, evidence from concept learning suggests that negative evidence even constrains the generation of hypotheses already at the outset of learning (Houtz, Moore, \& Davis 1973). Assuming a probability distribution over these hypotheses would imply an increase in probability of any of these hypotheses when excluding another hypothesis. In the previous example, introducing negative evidence that excludes the noise and music in general hypotheses would hence lead to an increase in likelihood for the remaining two hypotheses. Whether this increase is large enough to simulate our empirical findings depends on the prior probabilities of each of the hypotheses.

The question then becomes how to arrive at the right prior probabilities. The priors can be considered an implementation of prior knowledge that people have regarding the domain and feature that form the topic of the argument. Heit (1998) proposed that the prior probability for each hypothesis depends on the number of familiar properties that can be retrieved from memory, and have the same extension as the hypothesis proposes: The extension of a novel feature is likely if its distribution resembles that of many already-known properties. It is, however, not immediately obvious whether a process of sampling properties can result in priors that reflect the strong clustering of categories necessary to raise the argument strength with negative evidence.

Another fruitful approach to the question about priors has been provided by the structured statistical models approach (Kemp \& Tenenbaum, 2009; Tenenbaum, Kemp, $\&$ Shafto 2007). They propose that the priors for the Bayesian inference derive from a stochastic process that operates on a knowledge structure. The knowledge structure (e.g., a causal food web or a taxonomic tree) captures the structure in the world that is informative for a certain argument-for instance, the taxonomic relations for inferences regarding properties in animals. If we assume a similarity representation (a tree representation or a spatial representation) and a diffusion process that distributes a feature smoothly over the structure (Kemp \& Tenenbaum, 2009), as seems appropriate for the present task, the model predictions follow the Monotonicity Principle and hence cannot explain our present findings. ${ }^{7}$ This is not to say that no combination of knowledge structure and stochastic

\footnotetext{
${ }^{7}$ Also, contrary to the nonmonotonicity effect found in Medin et al. (2003) when adding positive evidence, varying sampling assumptions (strong or weak sampling, see Kemp \& Tenenbaum, 2009; Tenenbaum \& Griffiths, 2001) would not explain the present findings.
} 
process exists that is able to supply appropriate priors for raising argument strength with negative evidence. The structured statistical models approach is a framework that allows many specific instantiations.

\section{Relevance theory}

An approach that is not formalized but may provide a process account to explain the present finding is the relevance theory of induction (Medin et al., 2003). The basic idea is that distinctive properties of the premise categories highlight relevant dimensions for induction. These dimensions are then either reinforced (in case of a match) or undermined (in case of a mismatch) by comparing the premises with the conclusion. Negative evidence may work at this process of reinforcement or undermining. If people find a relevant dimension for induction (e.g., classical music) that is common to the positive premise and the conclusion, negative evidence can either undermine or reinforce the validity of the dimension. The validity of a dimension is undermined when negative evidence shares that dimension with the positive premise and the conclusion (e.g., "Haydn doesn't elicit alpha waves; thus, classical music cannot be the basis for induction") and reinforced when it does not share that dimension (e.g., "AC/DC does not elicit alpha waves, but $\mathrm{AC} / \mathrm{DC}$ is not classical music"). Whether negative evidence that reinforces a dimension is then considered relevant enough to increase argument strength depends on whether the negative evidence increases the salience of the dimension sufficiently above what it would have been without the negative evidence. In other words, the likelihood of a generalization from Shostakovich to Bach will increase with the introduction of negative evidence, if the negative evidence raises the salience of classical music as a basis for induction.

How might that happen? The relevance approach suggests that both the level of effort necessary to process an input, and the effect that such an input has, affect the relevance of the input (Sperber \& Wilson, 1995). Hence, if the negative evidence lowers the effort necessary to draw out the dimension used for induction, then inductive strength may increase. Furthermore, inductive strength may increase, if the introduction of negative evidence highlights a particular dimension that brings about a stronger effect than a dimension that had been considered before the introduction of negative evidence.

This proposal is clearly only a rough sketch of a possible mechanism by which negative evidence may increase argument strength. As was pointed out by Medin et al. (2003), the concepts of effect and effort are notoriously vague, hence making a formalization of the relevance approach a difficult task. However, the effect presented here, with its proposed mechanisms within the framework of relevance, offers another way to test the relevance account of induction. Future studies may want to test whether the introduction of negative evidence can indeed highlight a dimension for induction that would otherwise not have been considered. Likewise, it is an empirical question whether negative evidence can lower the effort necessary to identify a relevant basis for induction.

\section{Conclusion}

The present article provides empirical evidence for the idea that negative evidence can increase argument strength. These findings constitute a new phenomenon of categorybased property induction that models of induction need to be able to accommodate.

In their current form, there are only two models of induction that can explicitly incorporate negative evidence (Blok et al., 2007; Kemp \& Tenenbaum, 2009). Employing minimal assumptions, we have offered a brief overview of the main models and discussed whether or how they could incorporate our findings. Those models that are solely based on the underlying similarity relations between premises and conclusion have a hard time accounting for the increase in argument strength. Bayesian models are better able to accommodate the phenomenon. However, they require a specific distribution of prior probabilities across hypotheses in order to explain the effect. Thus, the onus is on them to provide a reasonable mechanism that would result in these priors. The relevance theory provides an intuitive process account of how the present effect may come about. However, relevance theory is only a framework account that is not formalized. The relatively vague concepts of effort and effect afford a larger degree of flexibility and can hence easily accommodate a range of phenomena.

Phenomena such as the one presented here provide the opportunity to challenge underlying assumptions of models of induction. Deriving mechanisms to accommodate these findings leads to new predictions that in turn provide tests of the models. The common goal of all models of induction is to provide a sensible way to define the relevance of evidence for a conclusion. The violation of the Monotonicity Principle for negative evidence constitutes a clear constraint on models of induction and again highlights the importance of getting a clearer grasp of what determines the relevance of evidence - be it positive or negative.

Author note This work was supported by an F+Fellowship awarded to D. Heussen by the University of Leuven. Inquiries concerning this article should be addressed to D. Heussen or W. Voorspoels, Department of Psychology, K.U. Leuven, Tiensestraat 102, 3000 Leuven, Belgium (e-mail: daniel.heussen@psy.kuleuven.be; wouter. voorspoels@psy.kuleuven.be). 


\section{Appendix}

Table 1 Items used in Experiments 1 and 3

\begin{tabular}{|c|c|c|c|c|c|c|c|}
\hline & Positive Premise & Negative Premise & Conclusion & Exp. $1 \mathrm{~N}=32$ & Exp. $3 \mathrm{~N}=14$ & Exp. 3 Positive & Exp. 3 Mixed \\
\hline \multirow[t]{15}{*}{ Target Items } & French wine, & $\neg$ Australian wine & $\rightarrow$ Italian wine & 0.16 & 0.14 & 6.43 & 6.36 \\
\hline & Rabbits, & $\neg$ Hedgehogs & $\rightarrow$ Squirrels & 0.25 & 0.29 & 7.00 & 6.79 \\
\hline & BMW, & $\neg$ Fiat & $\rightarrow$ Mercedes & 0.28 & 0.29 & 6.71 & 6.64 \\
\hline & Ducks, & $\neg$ Sparrows & $\rightarrow$ Swans & 0.38 & 0.36 & 7.07 & 7.79 \\
\hline & Flutes, & $\neg$ Guitars & $\rightarrow$ Trumpets & 0.47 & 0.29 & 5.36 & 6.07 \\
\hline & Rubens, & $\neg$ Dali & $\rightarrow$ Van Eyck & 0.50 & 0.21 & 4.79 & 4.50 \\
\hline & Actors, & $\neg$ Librarians & $\rightarrow$ Politicians & 0.50 & 0.43 & 4.86 & 5.86 \\
\hline & Freight ships, & $\neg$ Hovercrafts & $\rightarrow$ Cruise ships & 0.50 & 0.57 & 7.00 & 8.07 \\
\hline & Atlantic, & $\neg$ Lake Balaton & $\rightarrow$ Mediterranean & 0.50 & 0.29 & 5.71 & 6.36 \\
\hline & Window glass, & $\neg$ Bottle glass & $\rightarrow$ Car glass & 0.53 & 0.14 & 5.43 & 5.57 \\
\hline & Polar bears, & $\neg$ Bisons & $\rightarrow$ Penguins & 0.53 & 0.57 & 7.07 & 8.29 \\
\hline & Mozart, & $\neg \mathrm{AC} / \mathrm{DC}$ & $\rightarrow$ Bach & 0.59 & 0.36 & 6.43 & 7.07 \\
\hline & Moths, & $\neg$ Spiders & $\rightarrow$ Flies & 0.63 & 0.21 & 5.64 & 6.07 \\
\hline & LCD TVs, & $\neg$ Old TV sets & $\rightarrow$ Plasma screens & 0.69 & 0.57 & 6.64 & 7.86 \\
\hline & Strawberries, & $\neg$ Bananas & $\rightarrow$ Raspberries & 0.75 & 0.43 & 6.86 & 7.36 \\
\hline \multirow[t]{15}{*}{ Control Items } & Guitarists, & $\neg$ Bass guitarists & $\rightarrow$ Violinists & 0.06 & 0.00 & 7.71 & 4.50 \\
\hline & Laptop PCs, & $\neg$ Palmtop PCs & $\rightarrow$ Calculators & 0.06 & 0.14 & 5.50 & 3.57 \\
\hline & Air guns, & $\neg$ Sniper rifles & $\rightarrow$ Hunting rifles & 0.13 & 0.21 & 6.43 & 6.07 \\
\hline & Ants, & $\neg$ Termites & $\rightarrow$ Bees & 0.13 & 0.00 & 4.64 & 3.43 \\
\hline & Potatos, & $\neg$ Beets & $\rightarrow$ Carrots & 0.13 & 0.07 & 5.50 & 4.14 \\
\hline & Horses, & $\neg$ Cows & $\rightarrow$ Goats & 0.13 & 0.00 & 5.36 & 2.71 \\
\hline & Papayas, & $\neg$ Star fruits & $\rightarrow$ Mangos & 0.16 & 0.00 & 5.93 & 5.21 \\
\hline & F16s, & $\neg$ Concordes & $\rightarrow$ Boeings & 0.19 & 0.07 & 6.21 & 4.43 \\
\hline & Oaks, & $\neg$ Willows & $\rightarrow$ Beechs & 0.19 & 0.07 & 5.07 & 4.07 \\
\hline & Swordfish, & $\neg$ Rays & $\rightarrow$ Tunas & 0.19 & 0.00 & 5.00 & 4.21 \\
\hline & Storks, & $\neg$ Crows & $\rightarrow$ Gooses & 0.22 & 0.07 & 6.00 & 4.07 \\
\hline & Picasso, & $\neg$ Magritte & $\rightarrow$ Warhol & 0.28 & 0.07 & 5.43 & 3.93 \\
\hline & Lions, & $\neg$ Coyotes & $\rightarrow$ Crocodiles & 0.28 & 0.00 & 5.00 & 3.71 \\
\hline & Tripel beer, & $\neg$ Dubbel beer & $\rightarrow$ Duvel beer & 0.31 & 0.00 & 6.21 & 4.71 \\
\hline & Snakes, & $\neg$ Wasps & $\rightarrow$ Scorpions & 0.31 & 0.21 & 5.93 & 5.50 \\
\hline
\end{tabular}

Column headings "Experiment 1" and "Experiment 3" contain the proportion of people indicating a preference for arguments containing negative evidence. Column headings "positive" and "mixed" show the ratings of single positive and mixed premise arguments on a scale from 0 to 10

Table 2 Proportion of people preferring arguments containing negative evidence. Item scores for each condition in Experiment 2

\begin{tabular}{|c|c|c|c|c|c|c|c|}
\hline \multirow[t]{2}{*}{ Positive Premise } & \multicolumn{3}{|c|}{ Negative Premise } & \multirow[t]{2}{*}{ Conclusion } & \multirow[t]{2}{*}{ Target $\mathrm{N}=41$} & \multirow[t]{2}{*}{ Control $\mathrm{N}=41$} & \multirow[t]{2}{*}{ Irrelevant $\mathrm{N}=39$} \\
\hline & Target & Control & Irrelevant & & & & \\
\hline Rubens, & $\neg$ Dali & $\neg$ Van Gogh & $\neg$ Postmen & $\rightarrow$ Van Eyck & 0.37 & 0.22 & 0.23 \\
\hline Actors, & $\neg$ Librarians & $\neg$ Talk show hosts & $\neg$ Joists & $\rightarrow$ Politicians & 0.39 & 0.07 & 0.21 \\
\hline Freight ships, & $\neg$ Hovercrafts & $\neg$ Ferries & $\neg$ Volkswagen beetle & $\rightarrow$ Cruise ships & 0.41 & 0.17 & 0.31 \\
\hline Atlantic, & $\neg$ Lake Balaton & $\neg$ Indian Ocean & $\neg$ Mustard gas & $\rightarrow$ Mediterranean & 0.27 & 0.05 & 0.15 \\
\hline Window glass, & $\neg$ Bottle glass & $\neg$ Mirror glass & $\neg$ Screwdrivers & $\rightarrow$ Car glass & 0.34 & 0.34 & 0.28 \\
\hline Polar bears, & $\neg$ Bisons & $\neg$ Orcas & $\neg$ Woodlice & $\rightarrow$ Penguins & 0.54 & 0.27 & 0.33 \\
\hline Mozart, & $\neg \mathrm{AC} / \mathrm{DC}$ & $\neg$ Haydn & $\neg \mathrm{A}$ falling rock & $\rightarrow$ Bach & 0.66 & 0.10 & 0.44 \\
\hline Moths, & $\neg$ Spiders & $\neg$ Dragonflies & $\neg$ Lions & $\rightarrow$ Flies & 0.37 & 0.12 & 0.33 \\
\hline LCD TVs, & $\neg$ Old TV sets & $\neg$ Mobile screens & $\neg \mathrm{A}$ sheet of paper & $\rightarrow$ Plasma screens & 0.66 & 0.37 & 0.31 \\
\hline Strawberries, & $\neg$ Bananas & $\neg$ Brambles & $\neg$ Grass haulms & $\rightarrow$ Raspberries & 0.34 & 0.02 & 0.36 \\
\hline
\end{tabular}

Columns contain the proportion of people indicating a preference for arguments containing negative evidence in each condition. Sample size for each condition is given at the top 


\section{References}

Blok, S. V., Medin, D. L., \& Osherson, D. (2007). Induction as conditional probability judgment. Memory \& Cognition, 35, $1353-1364$.

Feeney, A. (2007). How many processes underlie category-based induction? Effects of conclusion specificity and cognitive ability. Memory \& Cognition, 35, 1830-1839.

Feeney, A., Coley, J., \& Crisp, A. (2010). The relevance framework for category-based property induction: Evidence from gardenpath arguments. Journal of Experimental Psychology. Learning, Memory, and Cognition, 36, 906-919.

Hampton, J. A., \& Cannon, I. (2004). Category-based induction: An effect of conclusion typicality. Memory \& Cognition, 32, 235-243.

Hayes, B., Heit, E., \& Swendsen, H. (2010). Inductive reasoning. Wiley interdisciplinary reviews: Cognitive science, 1, 278-292.

Heit, E. (1998). A Bayesian analysis of some forms of inductive reasoning. In M. Oaksford \& N. Chater (Eds.), Rational models of cognition (pp. 248-274). Oxford, England: Oxford University Press.

Heit, E. (2000). Properties of inductive reasoning. Psychonomic Bulletin \& Review, 7, 569-592.

Heit, E., Hahn, U., \& Feeney, A. (2004). Defending diversity. In W. Ahn, R. L. Goldstone, B. C. Love, A. B. Markman, \& P. Wolff (Eds.), Categorization inside and outside of the lab: Festschrift in honor of Douglas L. Medin. Washington, DC: APA Press.

Hempel, C. G. (1945). Studies in the logic of confirmation I. Mind, $54,1-96$.

Heussen, D., \& Hampton, J. A. (2011). Induction with mixed evidence: The role of typicality. Manuscript submitted for publication.

Heussen, D., Voorspoels, W., \& Storms, G. (2010). Can similaritybased models of induction handle negative evidence? In $\mathrm{S}$. Ohlsson \& R. Catrambone (Eds.), Proceedings of the 32nd Annual Conference of the Cognitive Science Society (pp. 20332038). Austin, TX: Cognitive Science Society.
Houtz, J. C., Moore, J. W., \& Davis, J. K. (1973). Effects of different types of positive and negative instances in learning "nondimensioned" concepts. Journal of Educational Psychology, 64, 206-211.

Kalish, C. W., \& Lawson, C. A. (2007). Negative evidence and inductive generalization. Thinking \& Reasoning, 13, 394-425.

Kemp, C., \& Tenenbaum, J. B. (2009). Structured statistical models of inductive reasoning. Psychological Review, 116, 20-58.

McDonald, J., Samuels, M., \& Rispoli, J. (1996). A hypothesis assessment model of categorical argument strength. Cognition, 59, 199-217.

Medin, D. L., Coley, J. D., Storms, G., \& Hayes, B. K. (2003). A relevance theory of induction. Psychonomic Bulletin \& Review, $10,517-532$.

Osherson, D. N., Smith, E. E., Wilkie, O., Lopez, A., \& Shafir, E. (1990). Category-based induction. Psychological Review, 97, 185-200.

Osherson, D. N., Stern, J., Wilkie, O., Stob, M., \& Smith, E. E. (1991). Default probability. Cognitive Science, 15, 251-269.

Rips, L. J. (1975). Inductive judgments about natural categories. Journal of Verbal Learning and Verbal Behavior, 14, 665-681.

Shepard, R. N. (1987). Toward a universal law of generalization for psychological science. Science, 237, 1317-1323.

Sloman, S. A. (1993). Feature-based induction. Cognitive Psychology, 25, 231-280.

Smith, E. E., Shafir, E., \& Osherson, D. N. (1993). Similarity, plausibility, and judgments of probability. Cognition, 49, 67-96.

Sperber, D., \& Wilson, D. (1995). Relevance: Communication and cognition (2nd ed.). Oxford, England: Blackwell.

Tenenbaum, J. B., \& Griffiths, T. L. (2001). Generalization, similarity, and Bayesian inference. The Behavioral and Brain Sciences, 24, 629.

Tenenbaum, J. B., Kemp, C., \& Shafto, P. (2007). Theory-based Bayesian models of inductive reasoning. In A. Feeney \& E. Heit (Eds.), Induction. Cambridge, UK: Cambridge University Press.

Waxman, S. R., Lynch, E. B., Casey, K. L., \& Baer, L. (1997). Setters and samoyeds: The emergence of subordinate level categories as a basis for inductive inference. Developmental Psychology, 33, $1074-1090$. 